\title{
p62 acts as an oncogene and is targeted by miR-124-3p in glioma
}

Danni Deng ${ }^{1,2+}$, Kaiming Luo ${ }^{2,3 \dagger}$, Hongmei Liu ${ }^{4 \dagger}$, Xichen Nie ${ }^{5}$, Lian Xue ${ }^{1,2}$, Rong Wang ${ }^{1,2}$, Yuan Xu ${ }^{1,2}$, Jun Cui ${ }^{5^{*}}$, Naiyuan Shao ${ }^{1 *}$ and Feng Zhi, ${ }^{1,2^{*}}$ (1)

\begin{abstract}
Background: Glioma is the most common central nervous system (CNS) tumour. p62, an important autophagy adaptor, plays a crucial role in cancer. However, the role of p62 in the progression of glioma is poorly characterized.

Methods: We examined the expression of p62 in glioma tissues and cell lines. Then we investigated the function of p62 in vitro, and clarified the mechanism underlying the regulation of p62 expression.

Results: We revealed that p62 was upregulated at both the mRNA and protein levels in human glioma tissues irrelevant to isocitrate dehydrogenase (IDH) status. Then, we found that overexpression of p62 promoted glioma progression by promoting proliferation, migration, glycolysis, temozolomide (TMZ) resistance and nuclear factor KB (NF-KB) signalling pathway, and repressing autophagic flux and reactive oxygen species (ROS) in vitro. In accordance with p62 overexpression, knockdown of p62 exerted anti-tumour effects in glioma cells. Subsequently, we demonstrated that miR-124-3p directly targeted the 3'-UTR of p62 mRNA, leading to the downregulation of p62. Finally, we found that p62 function could be partially reversed by miR-124-3p overexpression.
\end{abstract}

Conclusions: Our results demonstrate that p62 can be targeted by miR-124-3p and acts as an oncogene in glioma, suggesting the potential value of p62 as a novel therapeutic target for glioma.

Keywords: p62, miR-124-3p, Proliferation, TMZ resistance, Glycolysis, Migration

\section{Background}

As one of the most common and lethal central nervous system (CNS) tumours, gliomas are classified into four grades (I-IV) by the World Health Organization based on histopathology and molecular pathways [1]. Unfortunately, malignant gliomas (grade III to IV), which are associated with a dismal prognosis, constitute the

\footnotetext{
*Correspondence: cuij5@mail.sysu.edu.cn; naiyuanshao@126.com; danielzhif@suda.edu.cn

†Danni Deng, Kaiming Luo and Hongmei Liu contributed equally to this work

${ }^{1}$ Department of Neurosurgery, The First People's Hospital of Changzhou,

\#185 Juqian Road, Changzhou, Jiangsu, China

${ }^{2}$ Modern Medical Research Center, The Third Affiliated Hospital of Soochow

University, \#185 Juqian Road, Changzhou, Jiangsu, China

${ }^{5}$ MOE Key Laboratory of Gene Function and Regulation, State Key

Laboratory of Biocontrol, School of Life Sciences,

Sun Yat-sen University, \#135 Xingangxi Road, Guangzhou, China

Full list of author information is available at the end of the article
}

majority of gliomas. Currently, the standard treatment strategy for malignant glioma is maximal safe surgical resection followed by radiotherapy and/or adjuvant chemotherapy with temozolomide (TMZ). Despite recent clinical advances, the median survival time of patients diagnosed with malignant glioma is 1-2 years [2]. Hence, a deep understanding of the molecular mechanisms driving tumorigenesis and progression may provide candidates for more effective therapies for the treatment of malignant glioma.

p62, encoded by SQSTM1, is the most well-known autophagy adaptor, and it plays a crucial role in both normal physiology and cancer. Accumulating evidence demonstrates that aberrant p62 expression is associated with aggressive clinicopathologic features and poor prognosis in cases of pancreatic cancer, oral squamous cell carcinoma, metastatic breast cancer and hepatocellular carcinomas (HCC) [3-5]. p62 accumulation, induced by inflammation or autophagy 
impairment, promotes the initiation and progression of cancer through repressing apoptotic resistance and reactive oxygen species (ROS) generation and enhancing cell proliferation, survival, tumorigenesis and metastasis [4-6]. Additionally, high levels of p62 promote radiotherapy and chemotherapy resistance in hypopharyngeal carcinomas and ovarian cancer $[7,8]$. Research on p62 aberrance in the CNS has mainly focused on neurodegenerative disorders, such as Parkinson's disease and Alzheimer's disease, while the role of p62 in the progression of glioma is poorly characterized.

MicroRNAs (miRNAs) are endogenous, singlestranded, non-coding RNAs of approximately 19-25 nucleotides, and approximately $30 \%$ of protein-coding genes might be regulated by miRNAs [9]. MiRNAs can directly bind to complementary sequences in the mRNA 3'-UTR, resulting in translation inhibition or mRNA degradation [10]. MiR-124-3p, a brain-enriched miRNA, is either minimally expressed or absent and acts as a tumour suppressor in glioma, suggesting that miR-124-3p may be a novel diagnostic biomarker and therapeutic target in glioma [9]. Furthermore, miR124-3p can inhibit glioma progression through repressing proliferation, invasion and the stem-like traits, and potentiating chemosensitivity by targeting different genes $[11,12]$. In our previous work, we identified the anti-tumour effect of miR-124-3p in the pathogenesis of astrocytoma by targeting PIM1 [13].

Our study reveals the expression pattern and functions of p62 in glioma and further clarifies the regulatory mechanism between miR-124-3p and p62, suggesting the potential value of p62 as a therapeutic target for the treatment of glioma.

\section{Materials and methods Human tissues}

In total, 26 (normal tissue $=10$, grade $\mathrm{II}=4$, grade $\mathrm{III}=6$, grade IV $=6)$ tissue samples were collected from the Department of Neurosurgery at the Third Affiliated Hospital of Soochow University (Changzhou, China). Each patient signed an informed consent, and the study was approved by the Research Ethics Board of the Third Affiliated Hospital of Soochow University. Ten normal tissue samples were obtained from patients with cranial trauma resulting from traffic accidents. The patient cohort consisted of 9 males and 7 females. Of these patients, 10 were older than 50 years of age, while 6 were younger. Histological diagnoses were confirmed by three independent pathologists according to the WHO classification [1]. The tissues were immediately snap-frozen and stored in liquid nitrogen for further analysis.

\section{Cell lines and cell culture}

HEK293T, the human glial cell HEB and three glioma cell lines, including U87, LN229 and U251 were obtained from the Type Culture Collection of the Chinese Academy of Sciences (Shanghai, China). Cells were cultured in Dulbecco's Modified Eagle's medium (Gibco, USA) with $10 \%$ fetal bovine serum (FBS, Gibco, USA) at $37{ }^{\circ} \mathrm{C}$ with $5 \% \mathrm{CO}_{2}$.

\section{Cell transfection}

Synthetic miR-124-3p mimic (pre-miR-124-3p), mimic negative control (pre-ncRNA), miR-124-3p inhibitor (anti-miR-124-3p) and inhibitor negative control (antincRNA) were purchased from GenePharma (Shanghai, China).

Synthetic p62 siRNAs and negative control siRNA (nc-siRNA) were purchased from GenePharma (Shanghai, China). The sequences of these oligonucleotides are shown in Additional file 1: Table S1. The vector containing the full-length open reading frame (ORF) of p62 without its $3^{\prime}$-UTR and the negative control vector (ncvector) were purchased from Genewiz (Soochow, China).

All transient transfections were carried out with Lipofectamine $^{\mathrm{TM}} 2000$ Transfection Reagent (Invitrogen, USA) according to the manufacturer's specifications.

\section{Immunohistochemistry staining}

Immunohistochemical (IHC) analyses were performed to study p62 protein expression in the 26 tissues. The tissues were embedded in paraffin and then cut into $6-\mu \mathrm{m}$-thick sections. These sections were dewaxed in xylene and then rehydrated in an ethanol series. Pressure cooking in citrate buffer $(\mathrm{pH}=6)$ (Beyotime, China) for $5 \mathrm{~min}$ was applied to for antigen retrieval. Next, blocking of endogenous peroxidase was performed in $0.3 \% \mathrm{H}_{2} \mathrm{O}_{2}$ for $10 \mathrm{~min}$. Incubation with the p62 primary antibody (dilution 1:1000, AB56416, Abcam, USA) occurred overnight at $4{ }^{\circ} \mathrm{C}$. Detection was performed with HRP-labelled goat anti-mouse IgG (dilution 1:2000, A0216, Beyotime, China) for $30 \mathrm{~min}$, followed by incubation with the $D A B$ chromogen with a DAB horseradish peroxidase chromogen kit (Beyotime, China). Sections were counterstained in haematoxylin (Beyotime, China) for $10 \mathrm{~s}$ and dehydrated in an ethanol series.

\section{Mutation analysis of isocitrate dehydrogenase (IDH)}

Genomic DNA was extracted from frozen tissues using DNA extraction Kit (Tiangen, China), followed by quality evaluation on Nanodrop 1000 Spectrophotometer (Thermo Fisher, USA). The genomic regions which encompassed codons R132 of IDH1 and R172 of IDH2 were amplified by PCR and the sequences of primers are shown in Additional file 2: Table S2. Sanger sequencing 
reactions were performed by Genewiz, Inc. (Soochow, China).

\section{Fluorescence in situ hybridization analysis of $1 p / 19 q$ codeletion}

Four oligodendroglioma tissues were embedded in paraffin and then cut into $6-\mu \mathrm{m}$-thick sections $1 \mathrm{p} / 19 \mathrm{q}$ codeletion was detected with 1p36/1q25 and 19q13/19p13 Dual-Color Probe kit (Abbott Molecular Inc, USA) according to the manufacturer's specifications.

\section{RNA isolation and quantitative real-time PCR}

Total RNA was extracted from frozen tissues using TRIzol Reagent (Invitrogen, USA) according to the manufacturer's specifications. TaqMan miRNA probes (Thermo Fisher Scientific, USA) were used to quantify the level of miR-124-3p on the ABI 7500 System (Thermo Fisher Scientific, USA), as previously reported [13]. We used quantitative real-time PCR (qRT-PCR) to quantify the mRNA level of p62, C-C motif chemokine ligand 2 (CCL2), transforming growth factor beta 1 (TGF $\beta 1$ ), colony stimulating factor 3 (CSF3) and interleukin 6 (IL6) with SYBR Green PCR Master Mix (Takara, Japan) according to the manufacturer's instructions. U6 and $\beta$-actin served as the endogenous controls to miRNA and mRNA. The probes were purchased from Applied Biosystems (Thermo Fisher Scientific, USA), and the primers were purchased from Genewiz (Soochow, China). The sequences are shown in Additional file 2: Table S2.

\section{Western blot analysis}

Total protein was extracted from frozen tissues and western blot was carried out as previously described [13]. The following antibodies were used: p62 (dilution 1:1500, 5114), $\beta$-actin (dilution 1:3000, 3700), Lamin B1 (dilution $1: 1000,13,435)$, nuclear factor $\kappa B(N F-\kappa B)$ p65 (dilution 1:1000, 8242), HRP-labelled goat anti-mouse IgG (dilution 1:3000, A021) and HRP-labelled goat anti-rabbit IgG (dilution 1:3000, A0208). The yields of LC3 I and II was detected with LC3B antibody (dilution 1:2000, 3868). All the primary antibodies were purchased from Cell Signaling Technology (USA), and all the secondary antibodies were purchased from Beyotime (China). Protein bands were visualized with the ECL Advanced Western Blot Detection Kit (Thermo Fisher Scientific, USA) on the Bio-Rad ChemiDoc ${ }^{\mathrm{TM}}$ Touch (Bio-Rad, USA) and quantified with Image Lab (Bio-Rad, USA) software.

\section{Luciferase assay}

The wild-type 3'-UTR of the p62 luciferase reporter vector was constructed by amplifying the miR-124-3p binding sites in the p62 mRNA $3^{\prime}$-UTR and inserting them into the pMIR-REPORT ${ }^{\mathrm{TM}}$ plasmid (Thermo Fisher
Scientific, USA). A mutant vector was constructed to test for binding specificity. Next, $20 \mu \mathrm{g}$ reporter vectors and $10 \mu \mathrm{g}$ control Renilla luciferase plasmid pRL-SV40 (Promega, USA) were co-transfected into HEK293T cells $24 \mathrm{~h}$ before testing. The luciferase assay was performed using the Dual-Luciferase ${ }^{\circledR}$ Reporter Assay System (Promega, USA) according to the manufacturer's specifications.

\section{Wound healing assay}

The wound healing assay was carried out as previously reported [13]. At various time points, the plates were photographed with an IX71 microscope (Olympus, USA), and the distance was measured by ImageJ.

\section{Cell proliferation assay}

Transfected cells were seeded at 3000 cells per well in 96-well plates. Cell proliferation was assayed using CellCounting Kit 8 (CCK-8, Beyotime, China) at 0, 24, 48 and $72 \mathrm{~h}$, according to the manufacturer's specifications. The absorbance at a wavelength of $450 \mathrm{~nm}$ was measured with the BioTek Elx800 (BioTek, USA).

Relative TMZ toxicity was evaluated with a cell proliferation assay; $24 \mathrm{~h}$ after seeding, the cells were then treated with $400 \mu \mathrm{M}$ TMZ (Sigma, USA) or an equivalent solvent for $48 \mathrm{~h}$. After incubation with TMZ, the CCK-8 (Beyotime, China) assay was applied to investigate the relative TMZ toxicity.

\section{Migration assay}

As previously reported, transwell plates were used to assess cell migration in vitro [13]. The chambers were then photographed using an IX71 microscope (Olympus, USA) in six randomly selected fields. The number of migrated cells in every picture was counted.

\section{ROS assay}

Cellular ROS was assayed using a reactive oxygen species assay kit (Beyotime, China) according to the manufacturer's specifications and examined on the Guava EasyCyte 6HT-2L flow cytometer (Millipore, USA).

\section{Apoptosis assay}

Cells were incubated in $400 \mathrm{mM} \mathrm{TMZ}$ or an equivalent solvent for $48 \mathrm{~h}$. After incubation with TMZ, apoptosis was assayed with an Annexin V-FITC apoptosis detection kit (Beyotime, China) to investigate the effects of TMZ on cell apoptosis on the Guava EasyCyte 6HT-2L flow cytometer (Millipore, USA) according to the manufacturer's specifications. 


\section{Glycolysis assay}

The extracellular acidification rate (ECAR) was measured using a Seahorse XF-96 extracellular flux analyzer (Agilent, USA), and the experiment was carried out as previously described [13]. The ECAR was automatically calculated by Wave (Agilent, USA). Each data point represented the average of eight wells.

\section{Statistics}

All experiments were independently performed at least 3 times, and the means and standard error or standard deviation were subjected to the Student's $t$ test for pair wise comparison or ANOVA for multivariate analysis using GraphPad Prism software.

\section{Results}

\section{p62 is overexpressed in glioma}

To investigate the relationship between glioma malignancy and the expression of p62, we analysed the expression of p62 with IHC, western blot and qRT-PCR. First, we used qRT-PCR to qualify the expression of p62 mRNA in 10 normal tissues and 16 glioma tissues. 9 glioma tissues were IDH mutant and 7 were IDH wild-type. Detailed clinical and molecular characteristics of glioma patients were shown in Table 1. The relative mRNA levels of p62 were obviously upregulated in gliomas (Fig. 1a). Subsequently, we confirmed that accumulated p62 proteins were detected in the glioma samples, and p62 expression increased progressively from WHO grade II to IV (Fig. 1b-d). At last, we investigated the relative protein levels of p62 in human glial cell HEB and three glioma cell lines, including U87, LN229 and U251, and found p62 was overexpressed in glioma cell lines, compared with HEB (Fig. 1e). Furthermore, we found that

\section{Table 1 Clinical and molecular characteristics of glioma} patients

\begin{tabular}{llll}
\hline Parameters & WHO grade II & WHO grade III & WHO grade IV \\
\hline Number of patients & 4 & 6 & 6 \\
Gender & & & \\
Male & 2 & 3 & 3 \\
Female & 2 & 3 & 3 \\
Mean age (years) & $48.6 \pm 4.9$ & $49.3 \pm 3.8$ & $52.9 \pm 5.5$ \\
Histopathology & & 3 & \\
Astrocytoma & 3 & $3^{\text {a }}$ & 0 \\
Oligodendroglioma & 1 & 0 & 6 \\
$\quad$ Glioblastoma & 0 & & \\
IDH status & & 4 & 4 \\
Mutated & 3 & 2 & 4 \\
Wild type & 1 & & \\
\hline
\end{tabular}

a Two of the three oligodendroglioma tissues were $1 p / 19 q$ co-deleted expression of p62 had no correlation with IDH mutation status, indicating that the effect of p62 might not depend on IDH status (Additional file 3: Figure S1A, B). We decided to choose U87 and U251 glioma cell lines for further study. Our data suggest that p62 is closely involved in glioma progression and may be an oncogene in gliomas.

\section{p62 overexpression promotes cancer progression in glioma cells}

For the investigation of the function of aberrant p62 expression in glioma cells, p62 was overexpressed (Fig. 2a) by transiently transfecting U87 and U251 cells with the p62-overexpressing plasmid (p62-vector). The results of the cell proliferation assay indicated that p62 overexpression significantly promoted cell growth in U87 and U251 glioma cells (Fig. 2b). Since p62 is an important adaptor in autophagy, we treated transfected cells with chloroquine (CQ), an autophagy inhibitor, to evaluate the effect of p62 on autophagic flux. After incubation with $10 \mu \mathrm{M} \mathrm{CQ}$ for $24 \mathrm{~h}$, total protein was extracted for a western blot assay. The LC3 II/LC3 I ratio in the CQtreated p62-vector group was slightly lower than the CQtreated negative control vector (nc-vector) group in U87 cells, indicating that p62 overexpression could inhibit autophagic flux in U87 (Additional file 4: Figure S2A and $B$ ). Next, the wound healing assay was conducted to investigate the cell migration and invasion capabilities. The results showed that accumulation of p62 significantly increased the speed of wound closure in U87 and U251 cells (Fig. 2c). We then adapted a transwell assay to evaluate the role of p62 overexpression in cell migration. The overexpression of p62 markedly enhanced the migration capacity of U87 and U251 cells (Fig. 2d). Then we measured ECAR, an indicator of lactic acid production through glycolysis, to evaluate the effect of p62 overexpression on cellular energetics. A substantial promotion was observed in the p62-vector group, as shown by a significant increase in ECAR compared with that in the nc-vector group (Fig. 2e). Furthermore, the influence of p62 accumulation in TMZ resistance was investigated by CCK- 8 and cell apoptosis assays. Relative TMZ toxicity was significantly decreased in the p62-vector group compared with that in the nc-vector group (Fig. 2f). In accordance with the CCK-8 assay results, p62 overexpression could protect U87 and U251 cells from apoptosis induced by TMZ (Fig. 2g). Then we evaluated the effect of p62 overexpression on oxidative stress by measuring the reactive oxygen species in transfected cells, and the results showed that p62 accumulation caused mild decreases in the levels of reactive oxygen species (Fig. $2 \mathrm{~h}$ ). We also investigated the effects of p62 overexpression in NF- $\kappa B$ signalling pathway. The level of nuclear NF- $\kappa B$ 


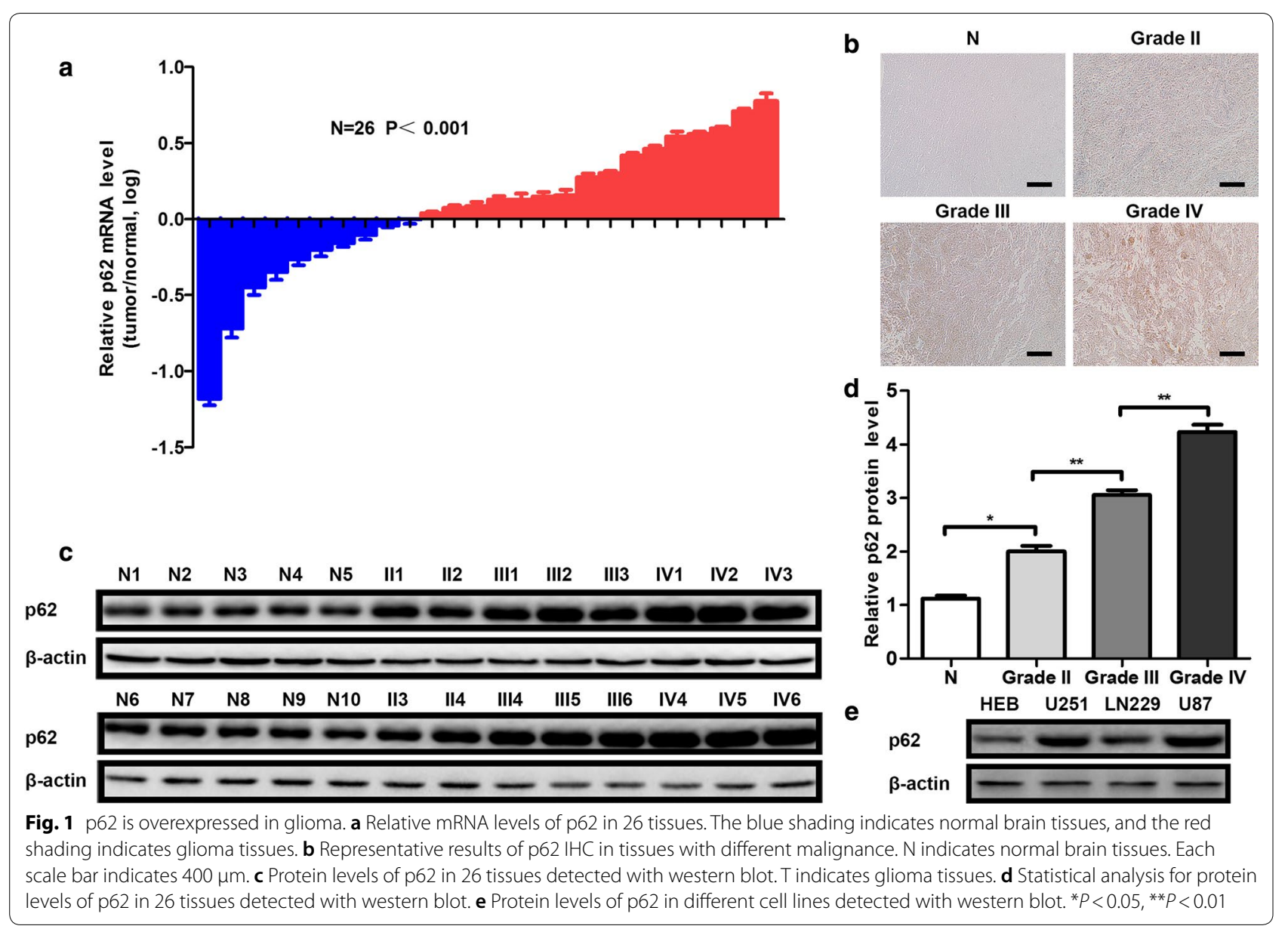

was slightly increased in p62 overexpression group, compared with nc-vector group (Additional file 4: Figure $\mathrm{S} 2 \mathrm{C})$. Several NF-kB downstream target genes including CCL2, IL-6, TGF 31 and CSF3 were examined. As shown in Additional file 4: Figure S2D-G, these genes were activated after p62 overexpression. Taken together, our data demonstrate that accumulated p62 can regulate cell proliferation, autophagy, wound healing, cell migration, glycolysis, TMZ resistance, oxidative stress and activate NF- $\mathrm{kB}$ signalling pathway, suggesting its oncogene role in gliomas.

\section{p62 knockdown represses cancer progression in glioma cells}

Given that p62 overexpression could promote glioma, we generated p62 knockdown (KD) cells with siRNAs to investigate whether downregulation of p62 could repress glioma cells. The western blot results showed that the siRNAs could knockdown the expression of p62 protein efficiently, especially p62 siRNA 2\# (Fig. 3a). CCK-8 results indicated that p62 knockdown significantly repressed cell growth (Fig. 3b). Next, the influence of p62 knockdown on autophagic flux was tested. There was no significant difference in the LC3 II/LC3 I ratio between the CQ-treated p62 KD group and the CQ-treated ncsiRNA group, indicating that p62 knockdown had no effect on autophagic flux in glioma cells (Additional file 5: Figure S3A, B). Subsequently, the migration and invasion capabilities were assessed by wound healing and transwell assay, and the results indicated that wound closure speed (Fig. 3c) and migration capacity (Fig. 3d) were significantly decreased in the p62 KD groups. Glycolysis was also measured, and there was a substantial block in glycolysis due to p62 knockdown (Fig. 3e). Next, TMZ resistance in p62 KD cells was evaluated. p62 KD cells were more sensitive to TMZ treatment (Fig. 3f, g). Finally, the reactive oxygen species were tested in p62 KD cells, and the results showed that p62 knockdown enhanced the levels of reactive oxygen species (Fig. 3h). Finally, as expected p62 knockdown inhibited nuclear NF- $\mathrm{B}$ expression and its downstream target genes (Additional file 5: Figure S3D-G). In sum, the loss of p62 functions opposite to p62 accumulation, thereby exerting antitumour effects in glioma. 

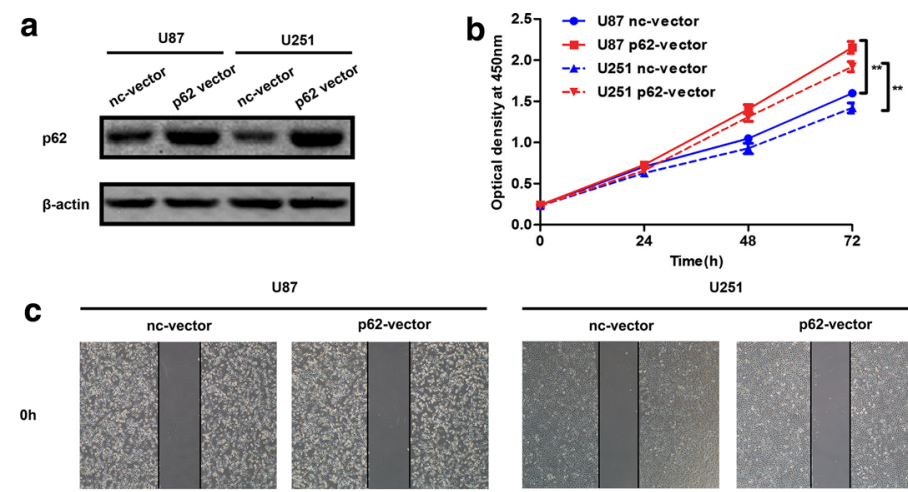

U87

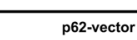

U251
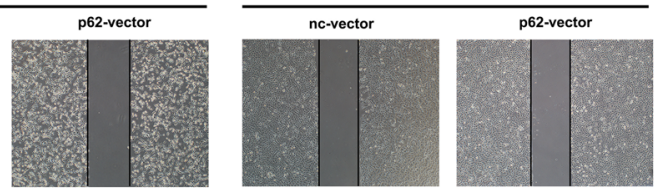

48h
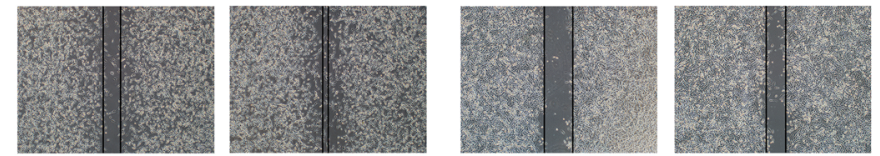

d

nc-vector p62-vector

U87

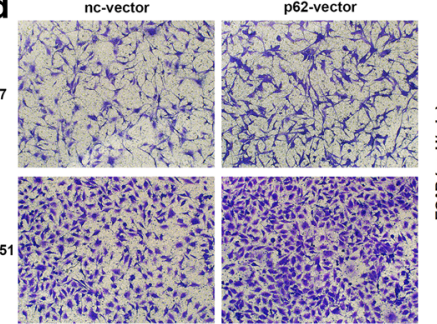

e
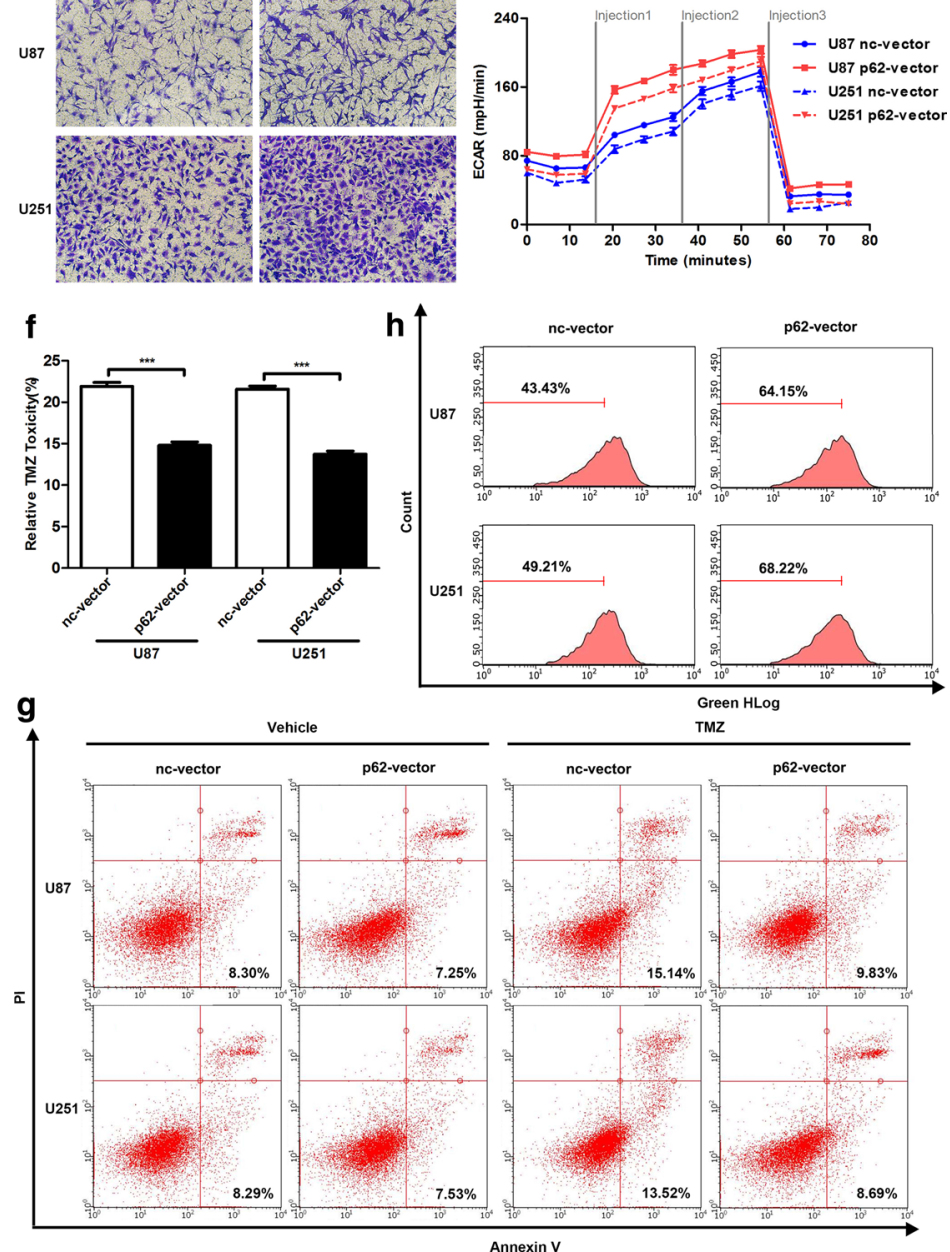

Fig. 2 p62 overexpression promotes cancer progression in glioma cells. a p62 protein levels in U87 and U251 cells after transfection with nc-vector or p62-vector detected with western blot. b Role of p62 overexpression in cell proliferation. $\mathbf{c}$ Representative results of wound healing assays in p62-overexpressed cells (×100 magnification). $\mathbf{d}$ Representative results of the transwell assay in p62-overexpressed cells (200 × magnification). e Role of p62 overexpression in glycolysis. $\mathbf{f}$ Role of p62 overexpression in TMZ resistance detected with CCK8 assay. $\mathbf{g}$ Representative results of apoptosis induced by TMZ in p62-overexpressed cells. $\mathbf{h}$ Representative results of ROS in p62-overexpressed cells. ${ }^{* *} P<0.01,{ }^{* * *} P<0.001$ 

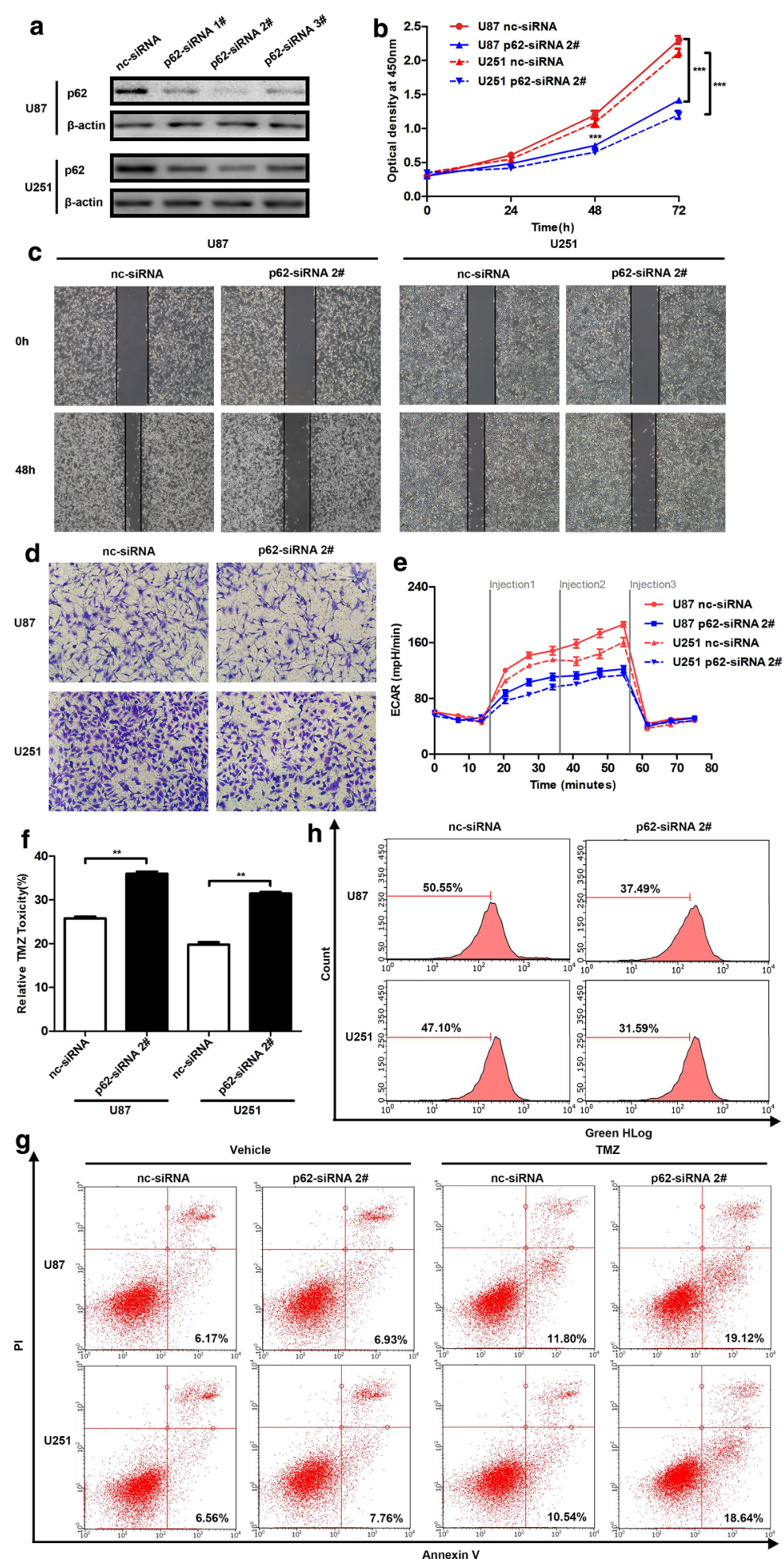

Fig. 3 p62 knockdown represses cancer progression in glioma cells. a Knockdown efficiency of p62 siRNAs in U87 and U251 cells detected with western blot. $\mathbf{b}$ Role of p62 knockdown in cell proliferation. c Representative results of wound healing assays in p62 knockdown cells ( $\times 100$ magnification). $\mathbf{d}$ Representative results of the transwell assay in p62 knockdown cells ( $\times 200$ magnification). e Role of p62 knockdown in glycolysis. $\mathbf{f}$ Role of p62 knockdown in TMZ resistance detected with CCK8 assay. $\mathbf{g}$ Representative results of apoptosis induced by TMZ in p62 KD cells. $\mathbf{h}$ Representative results of ROS in p62 knockdown cells. ${ }^{* *} P<0.01,{ }^{* * *} P<0.001$ 
p62 is directly targeted by miR-124-3p in glioma

We used TargetScan, a computational algorithm, to search for potential miRNAs targeting $\mathrm{p} 62$. The seed sequence of miR-124-3p matched 3'-UTR of p62, and the minimum free energy of the hybrids was $-13.3 \mathrm{kcal} / \mathrm{mol}$ (Fig. 4a). As previously reported, the expression of miR124-3p was significantly downregulated in glioma tissues, decreasing progressively from WHO grade II to IV (Fig. 4b) [13]. Furthermore, the relative levels of both p62 mRNA and protein increased as the relative miR-124-3p level decreased, suggesting that miR-124-3p might regulate p62 expression through both post-transcriptional mechanisms and mRNA stability (Fig. 4c, d).

For further investigation of the effect of miR-124-3p on p62 expression, miR-124-3p mimic and inhibitor were transfected into U87 and U251 cells. The expression of miR-124-3p significantly increased after transfection with pre-miR-124-3p and moderately decreased after transfection with anti-miR-124-3p
(Fig. 4e). To verify whether miR-124-3p affected p62 expression at both the mRNA and protein levels, we extracted the total RNA and protein contents of U87 and U251 cells and examined them with qRT-PCR and western blot, respectively, after transfection with pre-miR-124-3p and anti-miR-124-3p. MiR-124-3p overexpression significantly reduced the relative p62 mRNA level by approximately 65\%, and miR-124-3p inhibition increased the relative p 62 mRNA level by approximately $15 \%$ (Fig. 4f). The reduction in p62 protein level induced by miR-124-3p overexpression was approximately $45 \%$ (Fig. 4g). Since p62 was an important adaptor in autophagy and could be degraded along with autophagy, CQ was used to eliminate the potential influence of miR-124-3p on autophagic flux. The relative p62 protein level decreased with increases in the miR-124-3p dose, even in the CQ treatment group, indicating that miR-124-3p overexpression could repress the expression of p62 directly (Fig. 4h).

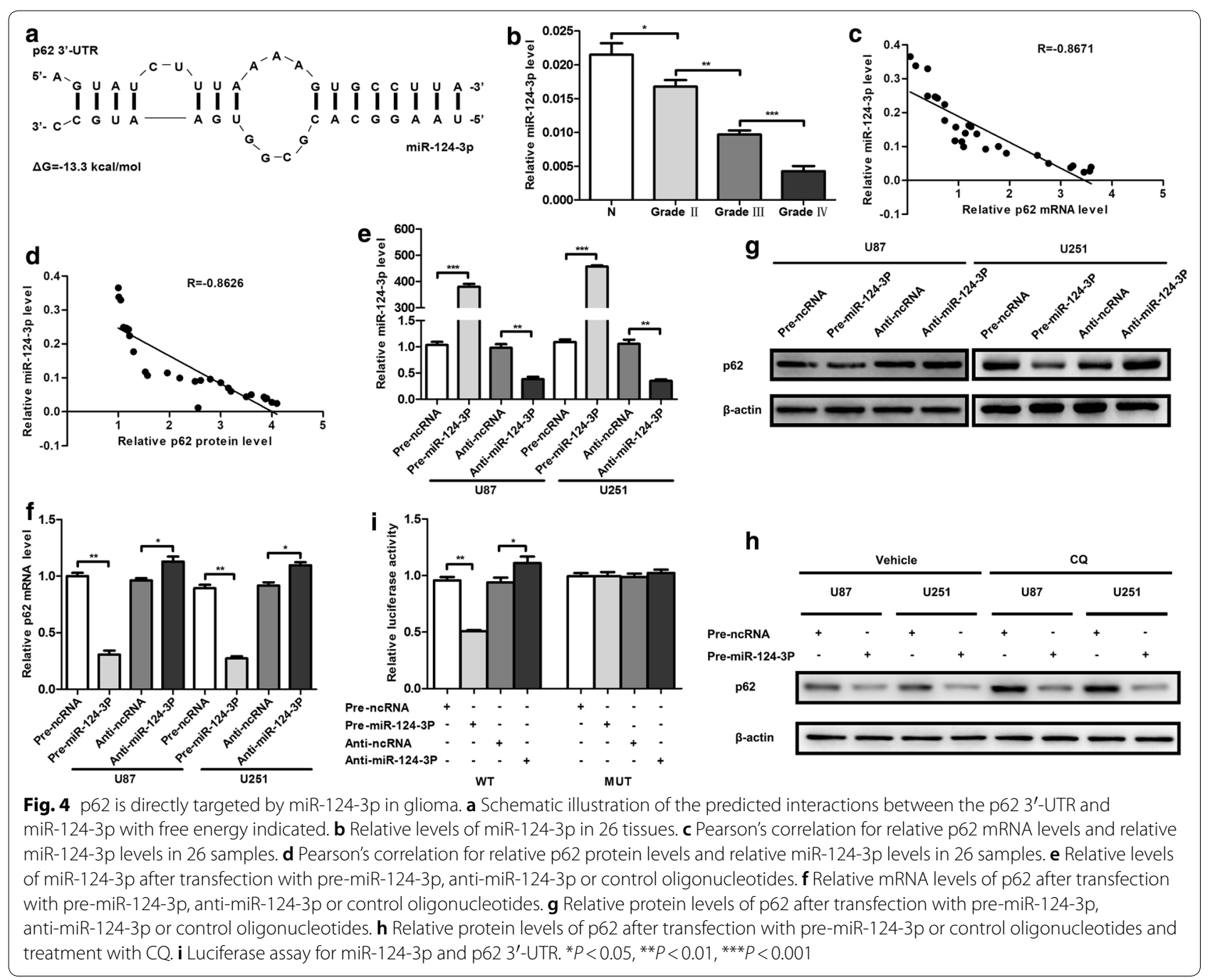


A luciferase assay was applied to investigate whether the influence of miR-124-3p on the expression levels of p62 was mediated through the predicted binding between miR-124-3p and mRNA 3'-UTR of p62. The results showed that pre-miR-124-3p could repress the luciferase activity compared with pre-ncRNA, while anti-miR-124-3p enhanced luciferase activity (Fig. 4i). The mutant firefly luciferase reporter plasmids, however, had no effect on luciferase activity in either the pre-miR124-3p or pre-ncRNA groups. These results indicate that the p62 mRNA $3^{\prime}$-UTR is directly targeted by miR124-3p, leading to the downregulation of p62 expression at both the mRNA and protein levels.

\section{p62 function is partially reversed by miR-124-3p}

Since the function of p62 and miR-124-3p was similar in U87 and U251, we investigated the influence of miR124-3p on p62 function in U87 glioma cell line only. Firstly, we used a western blot test to detect the integrative effect of p62 and miR-124-3p overexpression on the p62 protein level. p62 accumulation could be partially reversed by miR-124-3p overexpression (Fig. 5a). Then, we performed the CCK- 8 assay, the cell migration assay and the TMZ resistance assay to examine whether the introduction of miR-124-3p could reverse the functions of p62. The ectopic expression of miR-124-3p partially reversed the promotion of proliferation (Fig. 5b), migration (Fig. 5c) and TMZ resistance (Fig. 5d, e) induced by p62 overexpression, indicating that miR-124-3p exerted anti-tumour effects by suppressing p62 expression through binding to the p62 mRNA 3 '-UTR directly. We revealed the function of p62 in glioma cells and further examined the interaction between p62 and miR-124-3p to clarify the underlying mechanism of p62 in glioma.

\section{Discussion}

In this paper, p62 was identified as aberrantly upregulated at both the mRNA and protein levels in human glioma tissues irrelevant to IDH status. We then focused on the functions of p62 and demonstrated that the accumulation of p62 could promote glioma by regulating autophagy, proliferation, migration, reactive oxygen species, TMZ resistance, glycolysis and NF- $\mathrm{kB}$ signalling pathway. In accordance with p62 overexpression, p62 knockdown exerted anti-tumour effects in U87 and U251 glioma cells. Subsequently, we determined that miR-124-3p directly targeted the mRNA 3'-UTR of p62, leading to the downregulation of p62 expression at both the mRNA and protein levels. Furthermore, p62 function could be partially reversed by miR-124-3p overexpression. Our findings suggest the potential value of p62 as a novel therapeutic target for glioma. p62 is a multi-domain protein and thus exerts diverse functions through interacting with different molecules. First, p62 binds to mitogen-activated protein kinase kinase kinase 3 (MEKK3) through an N-terminal oligomerization domain (PB1), which leads to mTORC1 activation and c-Myc expression, thus promoting cancer cell proliferation in prostate cancer stromal fibroblasts and HCC $[5,14]$. In addition, p62 binds to LC3 on phagophore membranes through the LC3-interacting region (LIR) and delivers ubiquitylated cargos to the autophagosome for further degradation [15]. GATA4, a tumour suppressor, is degraded by p62-dependent selective autophagy and is confirmed to be downregulated in GBM [16]. Re-expression of GATA4 mediates sensitization of GBM cells to TMZ treatment through loss of APNG (alkylpurine-DNA- $N$-glycosylase), a poorly characterized DNA repair enzyme, instead of O-6-methylguanine-DNA-methyltransferase (MGMT) [17]. Moreover, p62 binds TNFR-associated factor 6 (TRAF6) with the TRAF6-binding domain (TB) to activate NF- $\kappa B$ signalling, resulting in the expression of inflammatory genes and cancer metastasis $[8,18]$. (4) Additionally, with the Keap1-interacting region (KIR), p62 binds to Kelchlike ECH-associated protein 1 (Keap1), leading to the activation of nuclear factor E2 related factor 2 (Nrf2), a transcription factor responsible for several antioxidant genes, which subsequently maintains a low level of ROS to protect cancer cells from oxidative damage $[5,6,19]$. Constitutive p62 and Nrf2 overexpression is detected in many tumour types and can favour cancer cell survival, promote cell proliferation and protect tumour cells from chemotherapy, radiotherapy and oxidative stress [6]. The overexpression of $\mathrm{Nrf} 2$ and p62 is observed in glioma samples and is identified to be closely related with the clinicopathological parameters and prognosis of patients with gliomas [20]. Finally, p62 knockdown cells exhibited decreased lactate secretion and glucose uptake through the mTORC1/c-Myc pathway and F1F0-ATP synthase dysfunction $[14,21]$. On the other hand, high p62 expression induces high hexokinase 2 and hypoxia-inducible factor $\alpha$ expression via the upregulation of mTORC1 and NF- $\kappa \mathrm{B}$ activity and the inhibition of von Hippel-Lindau E3 ubiquitin ligase activity, leading to enhancement of glycolysis in cancer [22, 23]. In our research, we have found that p62 acts as a tumour promotor by regulating autophagy, proliferation, migration, reactive oxygen species, TMZ resistance-, glycolysis and NF- $\mathrm{BB}$ signalling pathway in glioma cells. However, it has to be noted that the control for p62-overexpressing plasmid is an empty vector in our experiment which may cause the cells grow faster than other control, since DNA length can also affect the cell growth. This may be the reason that some difference in our assays are not so profound. The 

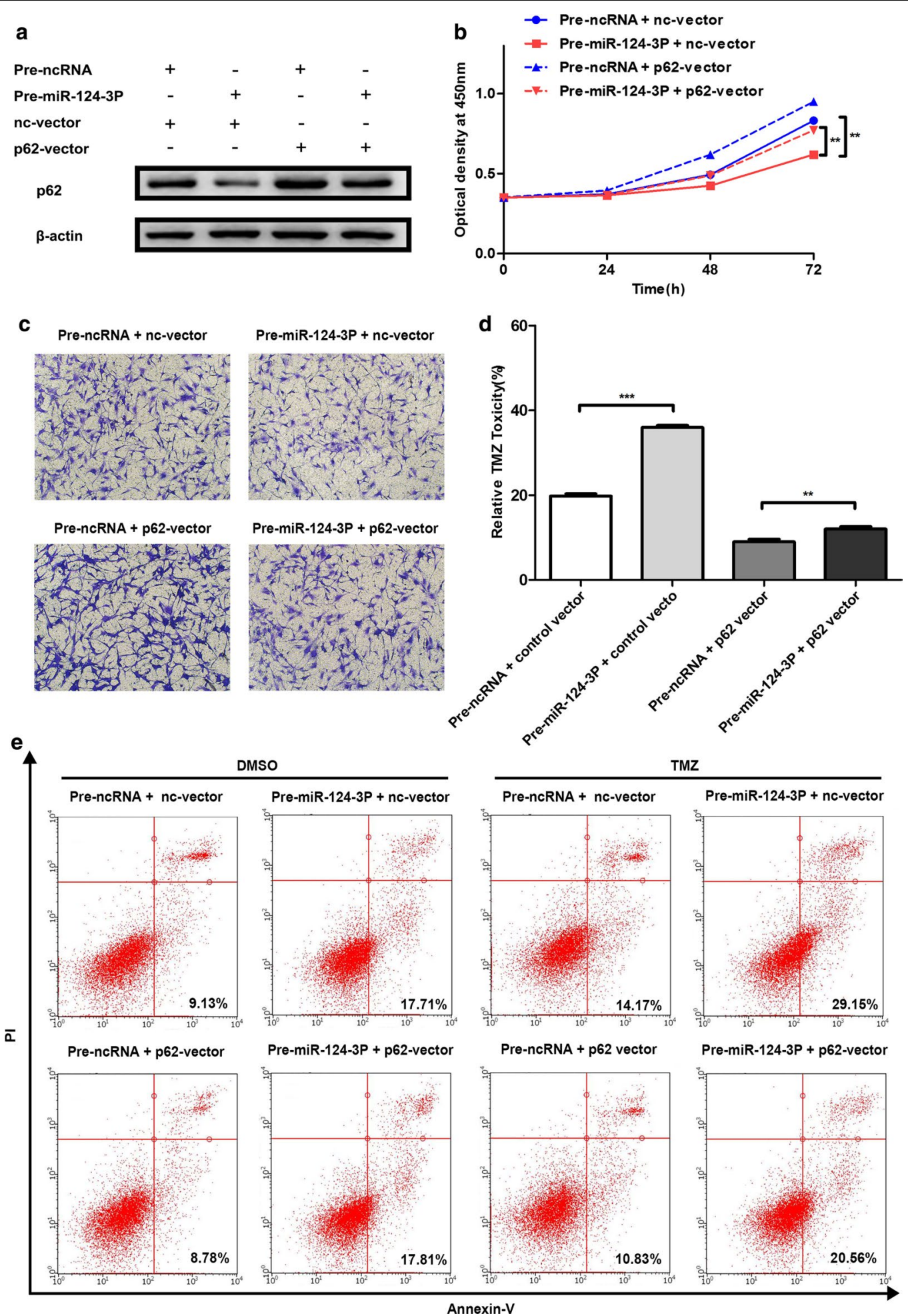

Fig. 5 p62 function is partially reversed by miR-124-3p. a Relative protein levels of p62 in U87 cells transfected with nc-vector or p62-vector, along with pre-ncRNA or pre-miR-124-3p. b Role of ectopic p62 and miR-124-3p expression in cell proliferation. c Role of ectopic p62 and miR-124-3p expression in cell migration (×200 magnification). $\mathbf{d}$ Role of ectopic p62 and miR-124-3p expression in TMZ resistance detected with CCK8 assay. $\mathbf{e}$ Representative results of apoptosis induced by TMZ in p62- and miR-124-3p-overexpressed cells. ${ }^{* *} P<0.01,{ }^{* * *} P<0.001$ 
expression of p62 can be regulated by various molecules with different mechanisms. Through the LC3-interacting region (LIR) domain, autophagy can bind p62 to LC3 on the membranes of autophagosomes and constantly degrade p62 via nonselective autophagy, thus playing a key role in the regulation of p62 levels [24]. Autophagy has routinely been suggested to potentiate the response to conventional therapies and inhibit tumour progression in gliomas [25]. 1L-6-hydroxymethylchiro-inositol 2(R)-2-O-methyl-3-O-octadecylcarbonate, an Akt inhibitor, reduces cell viability and radiosensitizes U87 glioma cells by inducing autophagy [26]. Imipramine can induce autophagy-associated cell death and reduce the incidence of gliomas in gliomagenesis models [27]. Gartanincan significantly induce autophagy and exhibit an anti-proliferation effect on T98G cells by inhibiting the PI3 K/ $\mathrm{Akt} / \mathrm{mTOR}$ signalling pathway [28]. Furthermore, NF- $\mathrm{kB}$, Nrf2and AP1 can induce SQSTM1 gene transcription, forming a positive feedback for p62 transcriptional regulation. As a result, inflammation and oxidative stress can induce p62 through NF- $\mathrm{kB}$ and Nfr2 to promote cell detoxification and selective autophagy, leading to the prevention of cell death and tumorigenesis [29]. Guanylatebinding protein3 (GBP3) promotes cell growth through activating the p62-ERK1/2 signalling pathway in glioma [30]. Furthermore, we found that there is no difference in the expression of p62 between IDH wild type group and IDH mutated group in our experiment, indicating that p62 function may be independent of IDH status. However, this still needs to be verified in future work. Additionally, p62 can be regulated by other molecules, such as microRNAs.

MiR-124-3p has been confirmed to be downregulated in glioma tissues, and loss of miR-124-3p is associated with high malignance and poor prognosis in patients with glioma [31, 32]. Furthermore, the relative levels of miR-124-3p in serum exosomes could serve as a complementary diagnostic biomarker, providing a minimally invasive and innovative tool to diagnose gliomas at their onset and predict metastases and glioma grading before surgery [33]. Because a single miRNA may target hundreds of mRNA targets, miR-124-3p exerts anti-tumour functions by suppressing a variety of target genes. MiR-124-3p inhibits glioma cell proliferation by blocking the expression of STAT3 [34], iASPP [35], Smad4 [36], and Nur77 [37]. MiR-124-3p restoration represses the migration and/or invasion of glioma cells by targeting Capn4 [38], IQGAP1 [39], iASPP [35], and PIM1 [13]. By directly targeting N-Ras and R-Ras, miR124-3p impairs angiogenesis through inhibiting VEGF transcription activation [40]. In this research, we demonstrate for the first time that miR-124-3p can directly regulate p62 in glioma. However, our experiment also has some flaws. The relatively low efficiency to inhibit miR-124-3p by anti-miR-124-3p may be the main reason why anti-miR-124-3p does not increase p62 levels considerably. In our future experiments, we will try to choose more efficient transfection tools to solve this problem.

\section{Conclusions}

In sum, p62 can be targeted by miR-124-3p and acts as a tumour promotor in glioma by regulating autophagy, proliferation, migration, reactive oxygen species, TMZ resistance, glycolysis and NF- $\mathrm{KB}$ signalling pathway in glioma cells, suggesting the potential value of p62 as a novel therapeutic target for glioma.

\section{Supplementary information}

Supplementary information accompanies this paper at https://doi. org/10.1186/s12935-019-1004-x.

Additional file 1: Table S1. Oligonucleotide sequences of siRNAs. Additional file 2: Table S2. qRT-PCR primers for amplification of IDH, miR124-3p, p62, CCL2, IL-6, TGF 31 and CSF3.

Additional file 3: Figure S1. Relationship between IDH mutation and the expression of p62. (A) Statistical quantitation of the p62 expression in IDH wildtype tumours vs IDH mutant tumours. (B) Statistical quantitation of the p62 expression in IDH wildtype tumours vs IDH mutant tumours with different malignance. ns indicates not significant.

Additional file 4: Figure S2. Role of p62 overexpression in cell autophagy and NF-KB signalling pathway. (A) Relative p62 protein levels in U87 and U251 cells after transfection with nc-vector or p62-vector and treatment with CQ. (B) Statistical quantitation of the role of p62 overexpression in cell autophagy detected with western blot. (C) Left: Nuclear NF-kB protein levels in U87 and U251 cells after transfection with nc-vector or p62-vector detected with western blot. Right: Statistical analysis. The relative protein expression of NF-KB in nc-vector transfected U87 or U251 cells were arbitrarily set as 1 . The results are presented as the mean \pm SD of three independent experiments. ( $D$ to $G$ ) Relative mRNA levels of CCL2, IL-6, TGF $\beta 1$ and CSF3 in p62-overexpressed cells. ${ }^{*} P<0.05,{ }^{* *} P<0.01$, ${ }^{* * *} P<0.001$, ns indicates not significant.

Additional file 5: Figure S3. Role of p62 knockdown in cell autophagy and NF-KB signalling pathway. (A) Relative p62 protein levels in U87 and U251 cells after transfection with nc-siRNA or p62-siRNAs and treatment with CQ. (B) Statistical quantitation of the role of p62 knockdown in cell autophagy detected with western blot. (C) Nuclear NF-kB protein levels in p62 KD cells detected with western blot. ( $D$ to $G$ ) Relative mRNA levels of CCL2, IL-6, TGF 31 and CSF3 in p62 KD cells. ${ }^{* *} P<0.01$, ${ }^{* *} P<0.001$, ns indicates not significant.

\section{Abbreviations}

CNS: central nervous system; TMZ: temozolomide; ROS: reactive oxygen species; 3'-UTR: 3'-untranslated region; qRT-PCR: quantitative real-time PCR; IDH: isocitrate dehydrogenase; CCL2: C-C motif chemokine ligand 2; TGF $\beta 1$ : transforming growth factor beta 1; CSF3: colony stimulating factor 3; IL-6: interleukin 6; NF-kB: nuclear factor KB; CCK-8: Cell-Counting Kit 8; ECAR: extracellular acidification rate; CQ: chloroquine; LIR: LC3-interacting region; GBM: glioblastoma multiform; Keap1: Kelch-like ECH-associated protein 1; Nrf2: nuclear factor $\mathrm{E} 2$ related factor 2.

Acknowledgements

Not applicable. 


\begin{abstract}
Authors' contributions
FZ, NYS and JC designed the experiments, supervised the study and revised the paper. DND, KML and HML completed the main experiments, analyzed the data and wrote the manuscript. XCN and LX helped with the main experiments; $Y X$ and RW were the major contributors in the clinical sample collection. All authors read and approved the final manuscript.

Funding

This work was supported by National Natural Science Foundation of China (81302197), Changzhou Science and Technology Support Program (CE20165048), Changzhou High-Level Medical Talents Training Project (2016CZBJ006), Changzhou Municipal Commission of Health and Family Planning Major Scientific and Technological Project (ZD201620), Changzhou Municipal Commission of Health and Family Planning Youth Talent Scientific and Technological Project (QN201807) and Natural Science Foundation of Jiangsu Province (BK20181156)
\end{abstract}

\section{Availability of data and materials}

The datasets analysed during the current available from the corresponding authors on reasonable request.

\section{Ethics approval and consent to participate}

Ethical approval was given by the Medical Ethics Committee of Soochow University.

\section{Consent for publication}

All authors are responsible for the submission of this article and accept the conditions of submission.

\section{Competing interests}

The authors declare that they have no competing interests.

\begin{abstract}
Author details
${ }^{1}$ Department of Neurosurgery, The First People's Hospital of Changzhou, \#185 Juqian Road, Changzhou, Jiangsu, China. ${ }^{2}$ Modern Medical Research Center, The Third Affiliated Hospital of Soochow University, \#185 Juqian Road, Changzhou, Jiangsu, China. ${ }^{3}$ Department of Endocrinology, The First People's Hospital of Changzhou, Changzhou, Jiangsu, China. ${ }^{4}$ School of Biological Science and Medical Engineering, Beihang University, \#37 Xueyuan Road, Beijing, China. ${ }^{5}$ MOE Key Laboratory of Gene Function and Regulation, State Key Laboratory of Biocontrol, School of Life Sciences, Sun Yat-sen University, \#135 Xingangxi Road, Guangzhou, China.
\end{abstract}

Received: 17 March 2019 Accepted: 28 October 2019

Published online: 06 November 2019

\section{References}

1. Bielle F. Building diagnoses with four layers: wHO 2016 classification of CNS tumors. Revue Neurologique. 2016;172(4-5):253-5.

2. Deshpande RP, Chandra Sekhar Y, Panigrahi M, Babu PP. SIRP alpha protein downregulates in human astrocytoma: presumptive involvement of HsamiR-520d-5p and Hsa-miR-520d-3p. Mol Neurobiol. 2017;54(10):8162-9.

3. Liu JL, Chen FF, Lung J, Lo CH, Lee FH, Lu YC, Hung CH. Prognostic significance of p62/SQSTM1 subcellular localization and LC3B in oral squamous cell carcinoma. Br J Cancer. 2014;111(5):944-54.

4. Li SS, Xu LZ, Zhou W, Yao S, Wang CL, Xia JL, Wang HF, Kamran M, Xue XY, Dong $L$, et al. p62/SQSTM1 interacts with vimentin to enhance breast cancer metastasis. Carcinogenesis. 2017;38(11):1092-103.

5. Umemura A, He F, Taniguchi K, Nakagawa H, Yamachika S, Font-Burgada J, Zhong Z, Subramaniam S, Raghunandan S, Duran A, et al. p62, upregulated during preneoplasia, induces hepatocellular carcinogenesis by maintaining survival of stressed HCC-initiating cells. Cancer Cell. 2016;29(6):935-48.

6. Son YO, Pratheeshkumar P, Roy RV, Hitron JA, Wang L, Zhang Z, Shi X. $\mathrm{Nrf2/p62} \mathrm{signaling} \mathrm{in} \mathrm{apoptosis} \mathrm{resistance} \mathrm{and} \mathrm{its} \mathrm{role} \mathrm{in} \mathrm{cadmium-}$ induced carcinogenesis. J Biol Chem. 2014;289(41):28660-75.

7. Arai A, Chano T, Ikebuchi K, Hama Y, Ochi Y, Tameno H, Shimada T. p62/ SQSTM1 levels predicts radiotherapy resistance in hypopharyngeal carcinomas. Am J Cancer Res. 2017;7(4):881-91.
8. Yan XY, Zhang Y, Zhang JJ, Zhang LC, Liu YN, Wu Y, Xue YN, Lu SY, Su J, Sun LK. p62/SQSTM1 as an oncotarget mediates cisplatin resistance through activating RIP1-NF-kappaB pathway in human ovarian cancer cells. Cancer Sci. 2017;108(7):1405-13.

9. Ye X, Wei W, Zhang Z, He C, Yang R, Zhang J, Wu Z, Huang Q, Jiang Q. Identification of microRNAs associated with glioma diagnosis and prognosis. Oncotarget. 2017;8(16):26394-403.

10. Anastasiadou E, Jacob LS, Slack FJ. Non-coding RNA networks in cancer. Nat Rev Cancer. 2018;18(1):5-18.

11. Xia H, Cheung WK, Ng SS, Jiang X, Jiang S, Sze J, Leung GK, Lu G, Chan DT, Bian XW, et al. Loss of brain-enriched miR-124 microRNA enhances stem-like traits and invasiveness of glioma cells. J Biol Chem. 2012;287(13):9962-71.

12. Qiao W, Guo B, Zhou H, Xu W, Chen Y, Liang Y, Dong B. miR-124 suppresses glioblastoma growth and potentiates chemosensitivity by inhibiting AURKA. Biochem Biophys Res Commun. 2017;486(1):43-8.

13. Deng D, Wang L, Chen Y, Li B, Xue L, Shao N, Wang Q, Xia X, Yang Y, Zhi F. MicroRNA-124-3p regulates cell proliferation, invasion, apoptosis, and bioenergetics by targeting PIM1 in astrocytoma. Cancer Sci. 2016;107(7):899-907.

14. Valencia T, Kim JY, Abu-Baker S, Moscat-Pardos J, Ahn CS, Reina-Campos M, Duran A, Castilla EA, Metallo CM, Diaz-Meco MT, et al. Metabolic reprogramming of stromal fibroblasts through p62-mTORC1 signaling promotes inflammation and tumorigenesis. Cancer Cell. 2014;26(1):121-35.

15. Mancias JD, Kimmelman AC. Mechanisms of selective autophagy in normal physiology and cancer. J Mol Biol. 2016;428(9):1659-80.

16. Kang C, Xu Q, Martin TD, Li MZ, Demaria M, Aron L, Lu T, Yankner BA, Campisi J, Elledge SJ. The DNA damage response induces inflammation and senescence by inhibiting autophagy of GATA4. Science. 2015;349(6255):5612.

17. Agnihotri S, Wolf A, Munoz DM, Smith CJ, Gajadhar A, Restrepo A, Clarke ID, Fuller GN, Kesari S, Dirks PB, et al. A GATA4-regulated tumor suppressor network represses formation of malignant human astrocytomas. J Exp Med. 2011;208(4):689-702.

18. Yang Q, Zhang MX, Zou X, Liu YP, You R, Yu T, Jiang R, Zhang YN, Cao $J Y$, Hong MH, et al. A prognostic bio-model based on SQSTM1 and $\mathrm{N}$-stage identifies nasopharyngeal carcinoma patients at high risk of metastasis for additional induction chemotherapy. Clin Cancer Res. 2018;24(3):648-58.

19. Hashimoto K, Simmons AN, Kajino-Sakamoto R, Tsuji Y, Ninomiya-Tsuji J. TAK1 regulates the Nrf2 antioxidant system through modulating p62/ SQSTM1. Antioxid Redox Signal. 2016;25(17):953-64.

20. Zhao M, Xu H, Zhang B, Hong B, Yan W, Zhang J. Impact of nuclear factor erythroid-derived 2-like 2 and p62/sequestosome expression on prognosis of patients with gliomas. Hum Pathol. 2015;46(6):843-9.

21. Galavotti S, Bartesaghi S, Faccenda D, Shaked-Rabi M, Sanzone S, McEvoy A, Dinsdale D, Condorelli F, Brandner S, Campanella M, et al. The autophagy-associated factors DRAM1 and p62 regulate cell migration and invasion in glioblastoma stem cells. Oncogene. 2013;32(6):699-712.

22. Chen K, Zeng J, Xiao H, Huang C, Hu J, Yao W, Yu G, Xiao W, Xu H, Ye Z. Regulation of glucose metabolism by p62/SQSTM1 through HIF1alpha. J Cell Sci. 2016;129(4):817-30.

23. Jiao L, Zhang HL, Li DD, Yang KL, Tang J, Li X, Ji J, Yu Y, Wu RY, Ravichandran $\mathrm{S}$, et al. Regulation of glycolytic metabolism by autophagy in liver cancer involves selective autophagic degradation of HK2 (hexokinase 2). Autophagy. 2017;1:14.

24. Choi J, Jung W, Koo JS. Expression of autophagy-related markers beclin-1, light chain 3A, light chain 3B and p62 according to the molecular subtype of breast cancer. Histopathology. 2013;62(2):275-86.

25. Palumbo $S$, Comincini S. Autophagy and ionizing radiation in tumors the "survive or not survive" dilemma. J Cell Physiol. 2012;228:1-8.

26. Fujiwara K, Iwado E, Mills GB, Sawaya R, Kondo S, Kondo Y. Akt inhibitor shows anticancer and radiosensitizing effects in malignant glioma cells by inducing autophagy. Int J Oncol. 2007;31(4):753-60.

27. Shchors K, Massaras A, Hanahan D. Dual targeting of the autophagic regulatory circuitry in gliomas with repurposed drugs elicits cell-lethal autophagy and therapeutic benefit. Cancer Cell. 2015;28(4):456-71.

28. Luo M, Liu Q, He M, Yu Z, Pi R, Li M, Yang X, Wang S, Liu A. Gartanin induces cell cycle arrest and autophagy and suppresses migration involving PI3 K/Akt/mTOR and MAPK signalling pathway in human glioma cells. J Cell Mol Med. 2017;21(1):46-57. 
29. Moscat J, Karin M, Diaz-Meco MT. p62 in cancer: signaling adaptor beyond autophagy. Cell. 2016;167(3):606-9.

30. Xu H, Sun L, Zheng Y, Yu S, Ou-Yang J, Han H, Dai X, Yu X, Li M, Lan Q. GBP3 promotes glioma cell proliferation via SQSTM1/p62-ERK1/2 axis. Biochem Biophys Res Commun. 2018;495(1):446-53.

31. Chen T, Wang XY, Li C, Xu SJ. Downregulation of microRNA-124 predicts poor prognosis in glioma patients. Neurol Sci. 2015;36(1):131-5.

32. Eguia-Aguilar P, Perezpena-Diazconti M, Benadon-Darszon E. Reductions in the expression of miR-124-3p, miR-128-1, and miR-221-3p in pediatric astrocytomas are related to high-grade supratentorial, and recurrent tumors in Mexican children. Child's Nervous Syst. 2014;30(7):1173-81.

33. Santangelo A, Imbruce P, Gardenghi B, Belli L, Agushi R, Tamanini A, Munari S, Bossi AM, Scambi I, Benati D, et al. A microRNA signature from serum exosomes of patients with glioma as complementary diagnostic biomarker. J Neuro-oncol. 2017;136(1):51-62.

34. Li W, Huang H, Su J, Ji X, Zhang X, Zhang Z, Wang H. miR-124 acts as a tumor suppressor in glioblastoma via the inhibition of signal transducer and activator of transcription 3. Mol Neurobiol. 2017:54(4):2555-61.

35. Liu X, Kang J, Sun S, Luo Y, Ji X, Zeng X, Zhao S. iASPP, a microRNA124 target, is aberrantly expressed in astrocytoma and regulates malignant glioma cell migration and viability. Mol Med Rep. 2018;17(1):1970-8.
36. Zhang Z, Gong Q, Li M, Xu J, Zheng Y, Ge P, Chi G. MicroRNA-124 inhibits the proliferation of C6 glioma cells by targeting Smad4. Int J Mol Med. 2017:40(4):1226-34.

37. Tenga A, Beard JA, Takwi A, Wang YM, Chen T. Regulation of nuclear receptor Nur77 by miR-124. PLoS ONE. 2016;11(2):e0148433.

38. Cai JJ, Qi ZX, Chen LC, Yao Y, Gong Y, Mao Y. miR-124 suppresses the migration and invasion of glioma cells in vitro via Capn4. Oncol Rep. 2016;35(1):284-90.

39. Lu SH, Jiang XJ, Xiao GL, Liu DY, Yuan XR. miR-124a restoration inhibits glioma cell proliferation and invasion by suppressing IQGAP1 and betacatenin. Oncol Rep. 2014;32(5):2104-10.

40. Shi Z, Chen Q, Li C, Wang L, Qian X, Jiang C, Liu X, Wang X, Li H, Kang C, et al. MiR-124 governs glioma growth and angiogenesis and enhances chemosensitivity by targeting R-Ras and N-Ras. Neuro-oncology. 2014;16(10):1341-53.

\section{Publisher's Note}

Springer Nature remains neutral with regard to jurisdictional claims in published maps and institutional affiliations.
Ready to submit your research? Choose BMC and benefit from:

- fast, convenient online submission

- thorough peer review by experienced researchers in your field

- rapid publication on acceptance

- support for research data, including large and complex data types

- gold Open Access which fosters wider collaboration and increased citations

- maximum visibility for your research: over $100 \mathrm{M}$ website views per year

At BMC, research is always in progress.

Learn more biomedcentral.com/submissions 